\title{
Mapping Behavioral Health Serious Game Interventions for Adults With Chronic Illness: Scoping Review
}

Teresa Hagan Thomas ${ }^{1}, \mathrm{PhD}, \mathrm{RN}$; Varshini Sivakumar ${ }^{1}, \mathrm{SN}$; Dmitriy Babichenko ${ }^{2}$, PhD; Victoria L B Grieve ${ }^{3}$, PharmD; Mary Lou Klem ${ }^{4}$, PhD, MLIS

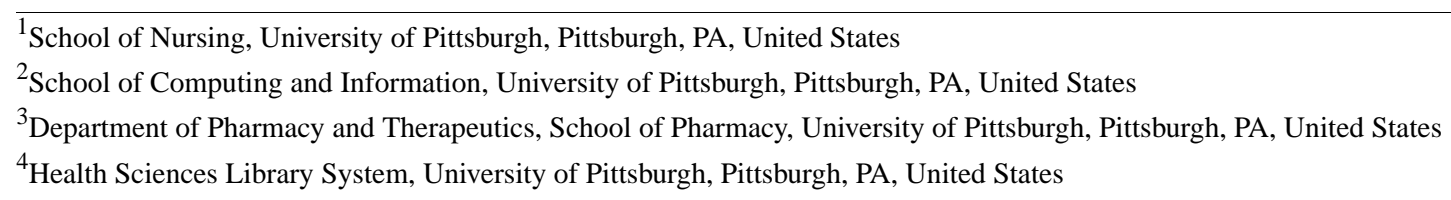

Corresponding Author:

Teresa Hagan Thomas, PhD, RN

School of Nursing

University of Pittsburgh

440 Victoria Building

3500 Victoria Street

Pittsburgh, PA, 15261

United States

Phone: 14126243799

Email: t.thomas@pitt.edu

\section{Abstract}

Background: Serious games for health are increasingly being used to address health outcomes in patients with chronic illnesses. These studies vary in their study designs, patient populations, frameworks, outcome variables, and degree of specificity of the serious game intervention.

Objective: This scoping review aims to clarify the conceptual features of the existing research related to serious games designed to improve cognitive and behavioral outcomes in adults with chronic illness.

Methods: We applied the Preferred Reporting Items of Systematic Reviews and Meta-Analysis for scoping reviews (PRISMA-ScR) methodology, including an a priori research question. We searched 4 electronic databases to identify articles published through November 2019. Inclusion criteria encompassed (1) adults 18 years or older; (2) patients with a diagnosis of chronic illness; (3) a serious game intervention; and (4) defined patient outcomes that assess patients' behavioral, cognitive, or health outcomes.

Results: Of the 3305 articles identified, 38 were included in the review. We charted and analyzed the theoretical frameworks, key concepts, and outcome variables of these studies with summaries of features across articles. The majority of studies used a randomized controlled trial design $(23 / 38,61 \%)$, included a custom serious game intervention $(22 / 38,58 \%)$, and lacked a theoretical framework $(25 / 38,66 \%)$. Common outcome variables included quality of life $(16 / 38,42 \%)$, mood $(15 / 38,39 \%)$, cognitive function $(13 / 38,34 \%)$, symptoms $(12 / 38,32 \%)$, and physical activity $(9 / 38,24 \%)$. Key differences between studies included whether or not serious games aimed to train versus teach patients, be widely accessible versus tailored interventions, or replace versus complement current treatments.

Conclusions: This scoping review defines the current landscape of research in serious games for health research targeting behavioral and cognitive outcomes in adults with chronic disease. Studies have addressed a variety of patient populations and diverse patient outcomes. Researchers wanting to build on the current research should integrate theoretical frameworks into the design of the intervention and trial to more clearly articulate the active ingredients and mechanisms of serious games.

(JMIR Serious Games 2020;8(3):e18687) doi: 10.2196/18687

\section{KEYWORDS}

review; chronic disease; behavioral sciences; video games 


\section{Introduction}

\section{Background}

Over the past decade, health-oriented clinical and research apps using electronic serious games have increased as a means to improve patient outcomes and provide health education [1]. Serious games take important health topics traditionally taught to patients and apply game features to provide a motivational, engaging, and even fun learning experience [2]. These games may be used to prevent disease [3], improve the health of patients with disease [4], and enhance social interaction to improve health [5]. Additional genres of serious games teach medical professionals skills [6-8], ultimately attempting to improve patient outcomes by improving clinician knowledge. A central attraction of electronic serious games is their ability to educate, motivate, and involve users without using conventional patient education that relies heavily on less engaging and often more intense training, such as written instruction or one-on-one consultation. The potential benefits of serious games include their high level of learning engagement, ease, and low cost of dissemination and distribution [9].

McGonigal [10], a game designer and leader in developing games to improve quality of life, defined serious games as having 4 hallmark features: (1) an overall goal-some desired outcome that provides a sense of purpose, (2) rules-limitations on the users' activities that necessitate creativity and strategic thinking, (3) feedback system - a way to communicate with users about their proximity to achieving a goal that motivates and promises that the goal is achievable, and (4) voluntary participation - the freedom to enter and leave the game so that participation is safe and pleasurable. The theorized learning mechanism leading serious games to be an effective teaching tool involves immersive qualities in which the users becomes engrossed in the game; the requirement for users to learn skills in increasingly difficult challenges; and use of the user's desire for mastery, arousal, diversion, and challenge [10,11].

Transformational games have emerged as a subset of serious games that try to positively impact the user by addressing outcomes, including behaviors, attitudes, and social issues [12]. Unlike traditional educational games that focus on the game as an end in itself, transformational games aim to have users learn through intentional participation in narratives that employ concepts that, if successfully learned, should extend beyond the game and meaningfully impact their lives [13]. This generalization requires that the game have specific behavior and learning outcomes, including an explicit plan for how users will transfer their skills from the game into real-world settings.

Rehabilitation and behavioral sciences have pioneered novel motivational, engaging games. Currently, the field is rapidly expanding to new patient populations and topics. Patients with chronic illnesses such as cancer, cardiovascular disease, diabetes, and obesity face long-term health problems that require diligent management. For these patients, behavioral, cognitive, and health outcomes are essential in ensuring that they can self-manage their illness and prevent long-term morbidity and mortality. These patient outcomes cut across different chronic diseases and include physical activity, maintenance of a healthy body weight, quality of life, symptom burden, mood, and cognitive function.

As electronic serious game interventions for health have increased, a lack of attention to the theoretical underpinnings has resulted in disparate game mechanisms, unclear theoretical frameworks of action, and weak study designs. Previous review articles have noted a need for improved research methods and expanded clinical applicability [14-17]. A fresh review of serious games in adults with chronic illnesses can assist researchers and clinicians in recognizing the strengths and limitations of how these studies have been designed. AA review can also identify ways to improve research rigor and translation to improved patient outcomes. Mapping the current research landscape will assist in establishing the key concepts, variables, theories, and frameworks undergirding this growing body of research.

\section{Objective}

Scoping reviews allow investigators to systematically examine emerging areas of evidence and can help identify gaps in knowledge, clarify concepts, and reveal methodological concerns in new areas of research. As scoping reviews generally have broader inclusion criteria than systematic reviews without an assessment of study quality, they allow for findings from disparate patient populations and contexts and a more comprehensive determination of evidence. Researchers and clinicians can apply the findings of a scoping review to more astutely build on the current evidence base and address gaps in existing research. Although systematic reviews of serious games have been conducted within specific patient populations and health care settings [18-21], the evidence supporting serious games focused on health skills and behaviors among adults with chronic diseases have not been mapped systematically. This scoping review aimed to define the concepts applied to studies using serious games to improve the health of adults with chronic illnesses.

\section{Methods}

\section{Research Question}

Our a priori research question was as follows: "What types of theoretical frameworks, key concepts, and outcome variables exist within serious game interventions to improve the cognitive and behavioral outcomes of adult patients with chronic illness?"

\section{Protocol}

We followed a scoping review methodology to synthesize concepts and research concerning the use of serious games as interventions designed to address cognitive and behavioral outcomes among adult patients with chronic illness. The objectives, inclusion criteria, and methods for this scoping review were specified in advance and documented in a protocol. Our protocol is freely available through the Open Science Framework [22]. This protocol follows the Preferred Reporting Items of Systematic Reviews and Meta-Analysis for scoping reviews (PRISMA-ScR) methodology to ensure that our results would be systematically conducted with minimal bias [23]. 


\section{Inclusion Criteria}

To be included in the scoping review, articles needed to focus on the following: (1) adults 18 years or older; (2) patients with a diagnosis of chronic illness; (3) a serious game intervention; and (4) defined patient outcomes that assess patients' behavioral, cognitive, and health outcomes.

\section{Participants}

We used definitions of chronic illness as defined by the Centers for Disease Control and Prevention [24] or the Center for Medicare and Medicaid Services [25]. These illnesses include Alzheimer's disease, arthritis, cancer, cardiovascular diseases including hypertension and stroke, chronic lung disease including asthma and chronic obstructive pulmonary disease (COPD), cystic fibrosis, diabetes, epilepsy and seizures, obesity, and oral health. Some articles included participants with illnesses that require long-term maintenance and ongoing medical care but were not listed in these definitions [26]. To err on the side of inclusion, we revised our definition of chronic illness to include additional diseases that could be considered chronic, such as Parkinson's disease. When studies included participants with and without chronic illnesses, we reviewed the full-text article to determine the proportion of participants with chronic illnesses and only included articles in which the majority of participants had an eligible disease.

\section{Concept}

We broadly defined serious games as "games that are designed to entertain players as they educate, train, or change behavior" [27]. We used the 4 criteria by McGonigal [10] listed above to determine whether an intervention included the requisite elements of a serious game. We did not stipulate that the serious game required any specified dose or intensity, length, or use of a comparison group. When limited descriptions of the game were available, we made assumptions about the existence of certain features to be liberal in article selection. Some articles included screenshots of the serious game, which indicated that the game included feedback, points, and engaging characters and scenery despite the article not describing these features. We did not include articles for which the serious game was not the intervention but was conceptualized as a diagnostic tool or priming event for a separate intervention. For example, some studies used video games to prime study patients' working memory and cognitive abilities, but the study was not designed to test the effect of that video game on patients' outcomes.

\section{Outcomes}

We included any study that assessed patients' behavioral, cognitive, and health outcomes. We created an ongoing list of outcome variables based on the variables identified in specific studies. Examples of behavioral outcomes included physical activity, medication adherence, and self-management.Examples of cognitive outcomes included executive function, working memory, learning new knowledge, and motivation. Examples of health outcomes included quality of life, mood, symptoms, BMI, hemoglobin $\mathrm{A}_{1 \mathrm{c}}\left(\mathrm{HbA}_{1 \mathrm{c}}\right)$, and blood pressure. We did not include functional outcomes (eg, the impact of a serious game on hand function or range of motion) because these assessments did not include the patient's involvement in learning or behaving to manage their chronic disease.

\section{Context}

As many interventions and meta-analyses have studied the impact of games to increase patients' mobility and functional outcomes [28] and do not include behavioral or cognitive outcomes, we only included serious game interventions that included behavioral, cognitive, or related health outcomes. For example, a study that analyzed the effect of a serious game on patients' motor control would not be included if the main outcomes only included functional measurements. A study would be included if the main outcomes included measures of patient self-management of their illness or symptoms.

\section{Identifying Relevant Studies and Study Selection}

The following databases were searched: PubMed (1946 to November 28, 2017), EbscoHost CINAHL (Cumulative Index of Nursing and Allied Health Literature; 1981 to November 2017), Ovid PsycINFO (1967 to November, Week 2, 2017), and EMBASE (Excerpta Medica dataBASE; 1974 to November 28, 2017). All database searches were run on November 28 , 2017, and, when available, a search limit to the English language was applied. Updated searches were run on November 25, 2019. An experienced health sciences librarian (MK) designed the PubMed search [22] and then translated that search for use in the other databases. The search strings consisted of natural language terms and (when available) controlled vocabulary representing the concepts of "videogames," "serious games," and "chronic diseases."

Two individuals independently screened abstracts and full-text articles using DistillerSR software (DistillerSR, Evidence Partners). This software allowed reviewers to collaboratively review all abstracts for the inclusion criteria. For all included articles, 2 reviewers extracted data and agreed upon the results. When reviewers disagreed on the inclusion criteria or data, they met to discuss the articles until consensus was reached.

Articles that met the inclusion criteria were charted to provide a descriptive summary. We extracted data including their study design; patient population; frameworks; behavioral, cognitive, or health-related outcomes; and a description of the serious game. We further described the key concepts that emerged as similarities and differences across the studies.

\section{Results}

The search strategy yielded 3268 references, of which 40 met the inclusion criteria. Figure 1 illustrates the PRISMA-ScR flowchart for our results. One research study had 2 manuscripts reporting different results from the same trial. As the framework, sample, and intervention were the same despite reporting on different outcome variables, we grouped these manuscripts as one study. A separate group of investigators had 2 manuscripts for the same trial and a separate manuscript reporting the study protocol. We grouped these manuscripts together, prioritizing the trial results. Our final sample included 38 studies. Table 1 illustrates the frequency of trial designs, populations, and most frequently cited study outcomes. 
Figure 1. Preferred Reporting Items of Systematic Reviews and Meta-Analysis for scoping reviews flowchart.

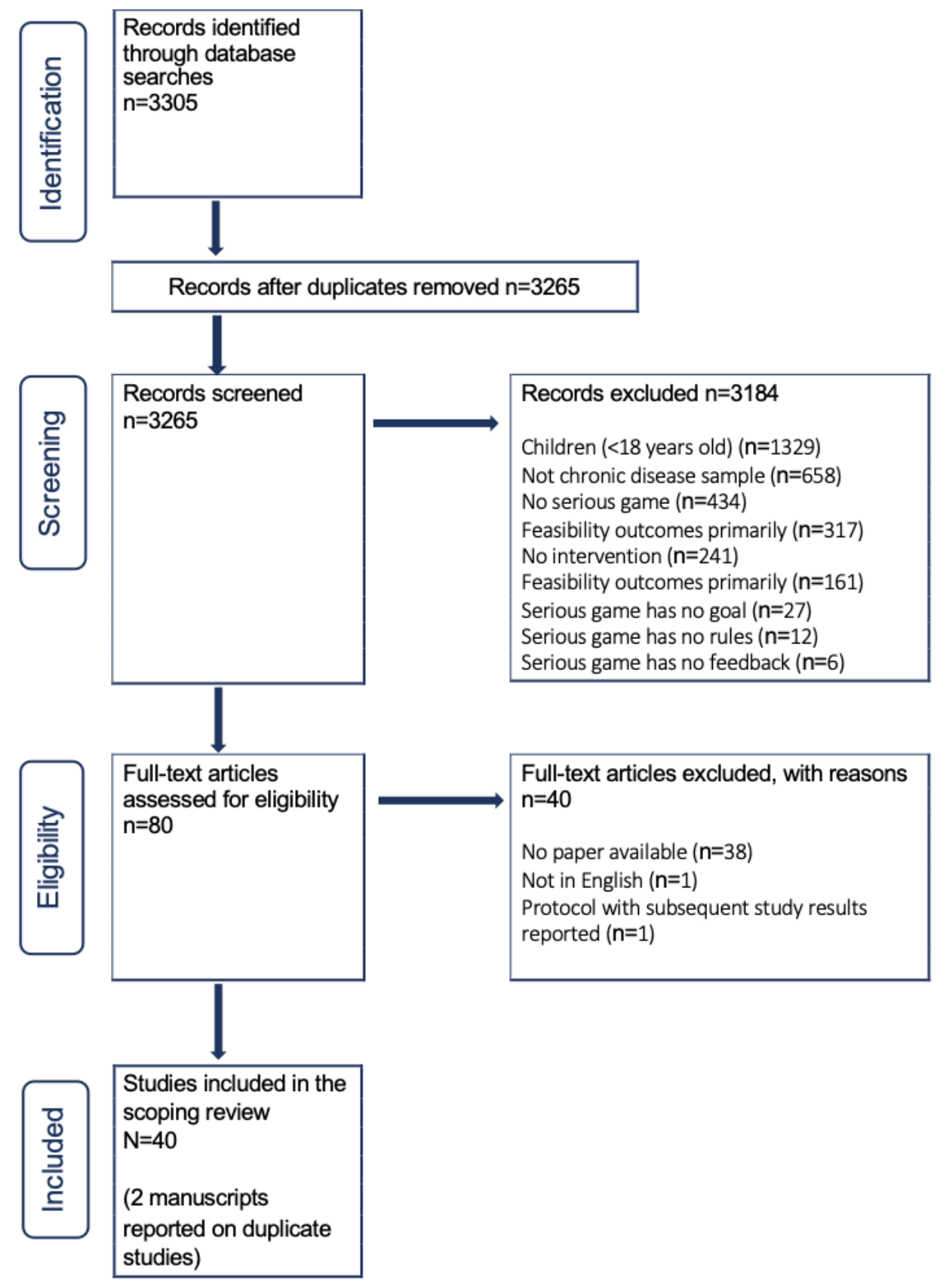


Table 1. Frequency of trial designs, patient populations, and study outcome variables.

\begin{tabular}{|c|c|}
\hline Characteristics & Values \\
\hline \multicolumn{2}{|l|}{ Trial design, $n(\%)$} \\
\hline Randomized controlled trial & $23(61)$ \\
\hline Feasibility study & $8(21)$ \\
\hline Protocol for study & $6(16)$ \\
\hline Case study & $1(3)$ \\
\hline \multicolumn{2}{|l|}{ Patient population, n (\%) } \\
\hline Stroke & $17(45)$ \\
\hline Cancer & $6(16)$ \\
\hline Diabetes & $5(13)$ \\
\hline Dementia & $3(8)$ \\
\hline Hypertension & $3(8)$ \\
\hline Parkinson's disease & $2(5)$ \\
\hline Obesity & $1(3)$ \\
\hline Chronic obstructive pulmonary disease & $1(3)$ \\
\hline \multicolumn{2}{|l|}{ Outcome variable, n (\%) } \\
\hline Quality of life & $16(42)$ \\
\hline Mood & $15(39)$ \\
\hline Cognitive function & $13(34)$ \\
\hline Symptoms & $12(32)$ \\
\hline Physical activity & $9(24)$ \\
\hline
\end{tabular}

\section{Trial Designs}

The majority of studies $(23 / 38,61 \%)$ reported the results of randomized controlled trials, whereas $16 \%$ (6/38) articles were protocols for clinical trials. Of these articles, the comparison groups for these trials mostly included a conventional form of active therapy $(23 / 29,79 \%)$, such as rehabilitation, rather than usual care or attention control $(6 / 29,21 \%)$. Few studies in the stroke population used a usual care comparison group, opting for conventional rehabilitation as a comparison. Less frequent trial designs included one-group pretest/posttest feasibility evaluations $(8 / 38,21 \%)$ and a case study $(1 / 38,3 \%)$.

\section{Populations}

The patient populations represented a diverse group of common chronic diseases, including stroke $(17 / 38,45 \%)$, cancer $(6 / 38$, $16 \%)$, diabetes $(5 / 38,13 \%)$, dementia $(3 / 38,8 \%)$, hypertension $(3 / 38,8 \%)$, Parkinson's disease $(2 / 38,5 \%)$, obesity $(1 / 38,3 \%)$, and COPD $(1 / 38,3 \%)$. The mean sample size was 82 patients (SD 104), with a range of 1 to 456. Most studies had small samples that were not adequately powered to test for statistically significant differences.

\section{Serious Games}

The majority of studies $(22 / 38,58 \%)$ described serious games that were custom-made interventions for specific patient populations. Studies describing custom-made games tended to have an in-depth description of the game's features, often because the research team built the game before evaluating it.
Three custom-made games used a version of the BrightArm virtual reality system [29-31]; all other games were evaluated in separate manuscripts.

The remaining studies $(16 / 38,42 \%)$ used off-the-shelf games designed for the general population. Off-the-shelf games included games within the Nintendo Wii Fit (11/38, 28\%), Xbox Kinect $(3 / 38,8 \%)$, and other off-the-shelf software $(2 / 38,5 \%)$. Often, the authors did not explicitly state the features of the off-the-shelf games. In lieu of such detail, we assumed that games such as bowling, tennis, and ping pong had a goal (score the most points), restrictions on actions (only specific actions are allowed), and feedback (points, badges, and scoreboards). Although these off-the-shelf games are commonly used as exercise, physical activity, and mobility interventions, different studies specified different rationales for the same technology. For example, different studies used the Nintendo Wii Fit game suite to improve patient motor function, balance, cognition, or general physical activity. Of the 17 studies reporting on patients recovering from a stroke, 8 (47\%) used off-the-shelf games.

\section{Theoretical Frameworks}

Most of the articles $(25 / 38,66 \%)$ lacked a clear theoretical framework. This was especially true for articles focused on patients with a history of stroke; only 3 of the 17 studies included a theoretical framework [31-33]. In most cases, the behavioral or cognitive outcomes appeared to serve as an add-on to other functional outcomes without an underlying hypothesis proposing how the serious game leads to improved outcomes. For example, authors may include measures of quality of life, 
depression, or motivation to their study but not indicate how these health outcomes related to the intervention.

Within the $34 \%(13 / 38)$ studies that cited a theoretical framework, frameworks varied in their application to the intervention and variables. Most frameworks focused on mechanics of specific interventions (simulation theory [33], cognitive training reorganization on brain network infrastructure [34], spaced education [35], neurofeedback for pain control [36], integrative rehabilitation [31], and self-management $[33,37])$. Other frameworks focused on intervention development (intervention mapping approach [38] and Gagne's instruction strategies [39]) and rationales for serious game features (behavioral economics [40,41], dual-task training [32], narrative transportation theory [42], self-determination theory [42-44], and behavior change theory $[43,44])$.

\section{Outcome Variables}

Studies varied greatly in the type and number of outcome variables used to assess the efficacy of the serious game. We report the most frequently cited outcome variables while noting several additional outcome variables. Multimedia Appendix 1 [29-68] includes a complete list of the behavioral, cognitive, and health outcomes as conceptualized within each study. We indicate whether the data for each outcome are based on patient self-report; examination with a valid assessment or clinical assessment; and/or objectively collected through sensor data, blood work, or medical records.

\section{Quality of Life}

Quality of life (including health-related quality of life) was the most common outcome across all studies (16/38; 42\%), including a variety of chronic diseases. Many studies included quality of life as an outcome, seeming to want to capture a patient's overall well-being. The rationale for including quality of life was rarely described. It was frequently captured as a secondary outcome to functional outcomes of mobility and strength in rehabilitation serious games for patients recovering from a stroke. Quality of life measures included self-report surveys, including the EuroQol-5 Dimension, Short Form Survey-36, and Stroke Impact Scale.

\section{Mood}

Mood-including depression and anxiety-was included as an outcome in $39 \%$ (15/38) studies. Similar to quality of life, mood variables were often included with minimal description tying these outcomes to the use and intent of the serious game intervention. Studies frequently included the Beck Depression Inventory as an outcome of interest, although the rationale for including this scale was lacking, and therefore, the discussion of these findings was minimal. Other studies measured patients' self-reported anxiety using the State-Trait Anxiety Inventory or disease-specific mood surveys.

\section{Cognitive Function}

Many serious games have been designed to improve memory and attention in patients with a history of stroke, dementia, and Parkinson's disease. Cognitive function was a common outcome in studies $(13 / 38,34 \%)$, especially for serious games targeting patients with dementia and stroke. Cognitive function assessments commonly included the Trail Making Test, Neuropsychological Assessment Battery, Mini-Mental Status Examination, and other neuropsychological evaluations that were administered by a trained study team member.

\section{Symptoms}

Physical symptoms such as fatigue and pain were reported as outcomes of the serious game in $32 \%$ (12/38) studies. Specific symptoms varied widely and were typically tailored to the patient population within the article (eg, fatigue was commonly assessed for patients with cancer $[38,42,49,50])$. Symptom measures included the Functional Assessment of Cancer Therapy, McGill Pain Questionnaire, and other self-report measures. Some studies measured symptoms using patient medical records that indicated symptom ratings and medication use to manage symptoms.

\section{Physical Activity}

A total of $24 \%$ studies (9/38) included a measure of physical activity as an outcome variable. In this case, physical activity was considered an activity of diabetes self-management, which would lead to better control of diabetes. Physical activity measures ranged from passive sensor data from wearable devices (eg, pedometers, accelerometers, and sensors on phones), clinical examinations of patients' cardiorespiratory fitness, to self-reported activity surveys.

\section{Other Outcomes}

Studies assessed myriad additional outcomes. Several studies assessed patients' motivation $(5 / 38,13 \%)$ in engaging in a serious game, captured by various self-report measures. Several studies assessed physical measurements conceptualized as health outcomes of desired behaviors. For example, studies targeting patients with diabetes often collected blood levels of $\mathrm{HbA}_{1 \mathrm{c}}$ $(4 / 38,11 \%)$ as an outcome variable indicating whether or not patients improved their average blood sugar level. Similarly, $3 \%$ study (1/38) assessed blood pressure as a target health outcome for patients with hypertension. Additional outcomes included patient self-reports of education or knowledge, self-management, medication adherence, and other outcomes. A minority of studies included outcomes related to learning within a serious game, fidelity of the intervention, or transference of learning within the game to real-life settings.

\section{Key Concepts}

\section{Teaching Versus Training}

A significant difference existed between the serious games that were described as teaching patients a specific behavior or skill compared with games intended to train patients in movements or activities. Games that were focused on teaching tended to have more explicit descriptions tying the features of the serious game to the intended learning outcomes, whereas games that were focused on training tended to have minimal explanation of how the game would train patients. Several studies employing a teaching approach cited theoretical frameworks linking how learning a behavior would lead to improved outcomes. For example, Kerfoot et al [35] explicitly stated how a spaced education and self-management framework would teach patients 
how to manage their diabetes, thereby reducing their $\mathrm{HbA}_{1 \mathrm{c}}$ and diabetes distress and increasing their knowledge of diabetes.

\section{Accessibility Versus Tailored}

Differences between the off-the-shelf and custom-made serious games was a consistent concept discussed across the studies. Many studies cited the advantages of using an off-the-shelf serious game such as Nintendo Wii Fit or Xbox Kinect, including easy integration into community settings and dissemination of effective protocols. Alternatively, studies in which the serious game was tailored to a specific patient population or health problem discussed the advantages of integrating specific content and mechanisms known to be important and relevant to that patient population. Tailoring may include specific technologies required to address specific health problems or a more general consideration of the psychosocial needs of the population. The Memory Matters game by Yu et al [68] intricately designed their interactive reminiscence game for patients with dementia, providing objects, images, and music familiar to the target patient population.

\section{Replacement Versus Complementary}

Several studies described the rationale for serious game intervention as a replacement for conventional treatment. In many cases, serious games were seen as a substitute for occupational or rehabilitation therapy in patients recovering from a stroke. Shin et al [61] created the RAPAEL Smart Glove to simulate upper extremity rehabilitation and compared it with conventional occupational therapy. This varied vastly from serious games meant to provide additional, novel, and meaningful support but otherwise not replacing an existing intervention, such as interventions focused on teaching self-management or coping skills. For example, Höchsmann et al $[43,44]$ reported on their intervention-MOBIGAME-which aimed to reduce diabetes by engaging patients in an immersive, relaxing program focused on increasing physical activity, motivation, and adherence.

\section{Discussion}

\section{Primary Findings}

This scoping review identified that electronic serious games for patients with chronic illness target a variety of patient populations, are mostly custom-made games, largely lack theoretical frameworks, and measure a broad array of patient outcomes. Common themes across studies included whether or not games were intended for teaching versus training purposes, meant to be widely available versus tailored to patient populations, and replace or complement existing therapies.

The 38 studies in this review represented 8 different patient populations, indicating that serious game interventions are applicable across diverse chronic illnesses. Many of the studies were designed for patients recovering from a stroke, possibly because off-the-shelf games (used in almost half of the studies for patients with stroke) included physical and functional targets similar to those used in conventional rehabilitation therapy. Nonetheless, a variety of serious games have been used across and within populations with chronic illness, underscoring the diversity of designs and settings in which researchers are investigating the efficacy of serious games.

A major limitation of the current landscape of serious games for adults with chronic illness is the overwhelming lack of theoretical frameworks. Two-thirds of studies did not cite a theoretical framework guiding the intervention, trial design, or proposed mechanisms linking the serious game to patient outcomes. This finding is similar to a recent systematic review of serious games [69]. One implication of this lack of frameworks is the broad list of study outcomes and lack of mechanistic or fidelity assessments. Outcomes were measured using diverse self-report measures, examinations, and sensor data, limiting the potential to compare outcomes across studies. The most frequently included outcomes, including quality of life and mood, were added without explicit hypotheses or proposed mechanisms linking them to the serious game. This finding corroborates other research [70], including a scoping review by Rohrbach et al [71], which found that virtual reality interventions for patients with stroke often include affective traits such as motivation and enjoyment without integration into the overall design and mechanistic planning of the intervention.

Recent consensus statements for the consistent integration of theoretical frameworks into serious games for health research provide researchers, developers, and clinicians with explicit recommendations on how to address this limitation of current studies [72]. Ideally, serious games for health would leverage the active ingredients of games (immersive, challenging, and chance for mastery) with persuasive strategies commonly used in behavior change research. Reassuringly, 5 of the $9(56 \%)$ studies published in 2019 cited a theoretical framework, perhaps indicating a trend in serious game research. In the future, research should integrate gamification and behavior change theory into rigorously designed trials based on theoretical frameworks [73].

Few studies have assessed the transfer of skills learned within a serious game into real-life settings. Although some studies assessed patient behaviors to determine whether the serious game led to changes in lifestyle and activity, this was infrequent and sometimes only assessed during the intervention period. A systematic review by Kuipers et al [74] noted a lack of attention to the mechanistic underpinnings of how serious games facilitate learning and drive their intended long-term effects. The authors urge researchers to consider game transference effects in the design of serious games for health, increasing the likelihood - or at least the ability to document - that these health interventions will lead to lasting desired patient behaviors and outcomes. Only one study addressed how a serious game was integrated into patient care via an existing research and clinical platform [40]. Researchers designing custom serious games for health should include upfront plans for disseminating their intervention within clinical care or broadly into larger populations.

The outcome variables of the studies included were inconsistently aligned with the serious game's goals. For example, games designed to improve cognition and physical fitness measured outcomes of executive function and physical activity, respectively. On the other hand, many studies included outcomes that were less clearly aligned with the intervention. 
This was especially true for studies that measured quality of life and mood. Many of these studies did not indicate a rationale for why quality of life or mood would change because of the serious game but appeared to be included as measures of patients' general well-being.

This scoping review has limitations. First, this review relied on published manuscripts of electronic serious games. We attempted to include a broad definition of both electronic serious games and chronic illnesses, although fluctuations in the terminology around these terms limited our search strategies' abilities to identify articles. Conference proceedings did not include sufficient detail to answer our research questions. Analog serious games were not included in this review and could be considered in future analyses. Second, this review relied on descriptions of serious games and interventions described within manuscripts, which were often limited, especially concerning game features and frameworks. Although we attempted to use our best judgment, additional details of the games and interventions could provide a more comprehensive summary. Finally, the broad nature of this review-although appropriate for a scoping review-limits the ability to provide specific conclusions based on patient population, game design, patient outcomes, etc. Future research could include more broader definitions of serious games, request information from authors to clarify game features and frameworks, and assess the efficacy of the serious game.

\section{Conclusions}

This review assists researchers in creating serious game interventions to address chronic health conditions by providing clarity on how to build from the current structure of serious games for health research. Researchers should continue the existing momentum in building robust, large trials driven by theoretical underpinnings of how interventions are hypothesized to impact outcomes. As attention to how game features lead to behavioral, cognitive, and health outcomes increases, we as a field will grow in the ability of our research to effectively and efficiently impact the most significant health problems patients experience.

\section{Acknowledgments}

This study was funded by the American Cancer Society Mentored Research Scholar Grant (MRSG-18-051-01) and the National Palliative Care Research Center Career Development Award.

\section{Authors' Contributions}

TT was the principal investigator. TT was involved in study conception, design, data capture, data analysis, and interpretation of the results and was the primary writer of the manuscript. VS was involved in data capture, data analysis, and editing of the manuscript. DB was involved in study conception, data analysis, and editing of the manuscript. VG was involved in study conception, data analysis, and editing of the manuscript. MK was involved in study conception, data capture, data analysis, and editing of the manuscript.

\section{Conflicts of Interest}

None declared.

\section{Multimedia Appendix 1}

Results table of studies of serious games for adults with chronic disease targeting behavioral, cognitive, or other health outcomes $(\mathrm{N}=38)$.

[DOCX File, 35 KB-Multimedia Appendix 1]

\section{References}

1. Sharifzadeh N, Kharrazi H, Nazari E, Tabesh H, Khodabandeh ME, Heidari S, et al. Health education serious games targeting health care providers, patients, and public health users: scoping review. JMIR Serious Games 2020 Mar 5;8(1):e13459 [FREE Full text] [doi: 10.2196/13459] [Medline: 32134391]

2. Garris R, Ahlers R, Driskell JE. Games, motivation, and learning: a research and practice model. Simul Gaming 2016 Aug 18;33(4):441-467. [doi: 10.1177/1046878102238607]

3. Ruiz-López T, Sen S, Jakobsen E, Tropé A, Castle PE, Hansen BT, et al. FightHPV: design and evaluation of a mobile game to raise awareness about human papillomavirus and nudge people to take action against cervical cancer. JMIR Serious Games 2019 Apr 8;7(2):e8540 [FREE Full text] [doi: 10.2196/games.8540] [Medline: 30958271]

4. Krebs P, Burkhalter J, Fiske J, Snow H, Schofield E, Iocolano M, et al. The QuitIT coping skills game for promoting tobacco cessation among smokers diagnosed with cancer: pilot randomized controlled trial. JMIR Mhealth Uhealth 2019 Jan 10;7(1):e10071 [FREE Full text] [doi: 10.2196/10071] [Medline: 30632971]

5. Santos LH, Okamoto K, Funghetto SS, Cavalli AS, Hiragi S, Yamamoto G, et al. Effects of social interaction mechanics in pervasive games on the physical activity levels of older adults: quasi-experimental study. JMIR Serious Games 2019 Jul 22;7(3):e13962 [FREE Full text] [doi: 10.2196/13962] [Medline: 31333202]

6. Klincova M, Harazim H, Schwarz D, Kosinova M, Smekalova O, Stourac P. What can be achieved with motivation-based teaching of medical students? A monocentric retrospective audit of retention among highly motivated graduates who 
underwent the learning-by-doing concept in anesthesiology and intensive care medicine. JMIR Serious Games 2019 Apr 9;7(2):e10155 [FREE Full text] [doi: 10.2196/10155] [Medline: 30964443]

7. Schwarz D, Štourač P, Komenda M, Harazim H, Kosinová M, Gregor J, et al. Interactive algorithms for teaching and learning acute medicine in the network of medical faculties MEFANET. J Med Internet Res 2013 Jul 8;15(7):e135 [FREE Full text] [doi: 10.2196/jmir.2590] [Medline: 23835586]

8. Chon S, Timmermann F, Dratsch T, Schuelper N, Plum P, Berlth F, et al. Serious games in surgical medical education: a virtual emergency department as a tool for teaching clinical reasoning to medical students. JMIR Serious Games 2019 Mar 5;7(1):e13028 [FREE Full text] [doi: 10.2196/13028] [Medline: 30835239]

9. Hamari J, Shernoff DJ, Rowe E, Coller B, Asbell-Clarke J, Edwards T. Challenging games help students learn: an empirical study on engagement, flow and immersion in game-based learning. Comput Hum Behav 2016 Jan;54:170-179. [doi: 10.1016/j.chb.2015.07.045]

10. McGonigal J. Reality Is Broken: Why Games Make Us Better and How They Can Change the World. New York, USA: Penguin Press; 2011.

11. Annetta LA. The 'I's' have it: a framework for serious educational game design. Rev Gen Psychol 2010 Jun;14(2):105-113. [doi: $10.1037 / \mathrm{a} 0018985$ ]

12. Kapp K. The Gamification of Learning and Instruction: Game-Based Methods and Strategies for Training and Education. San Francisco, CA: John Wiley \& Sons; 2012.

13. Culyba S. Transformational Games: A Process Tool for the Development of Transformational Games. Halifax, NS: ETC Press; 2018.

14. Barab S, Gresalfi M, Dodge T, Ingram-Goble A. Narratizing disciplines and disciplinizing narratives: games as 21st century curriculum. Int J Gaming Comput Mediat Simul 2010;2(1):17-30. [doi: 10.4018/jgcms.2010010102]

15. DeSmet A, van Ryckeghem D, Compernolle S, Baranowski T, Thompson D, Crombez G, et al. A meta-analysis of serious digital games for healthy lifestyle promotion. Prev Med 2014 Dec;69:95-107 [FREE Full text] [doi: 10.1016/j.ypmed.2014.08.026] [Medline: 25172024]

16. Johnson D, Deterding S, Kuhn K, Staneva A, Stoyanov S, Hides L. Gamification for health and wellbeing: a systematic review of the literature. Internet Interv 2016 Nov;6:89-106 [FREE Full text] [doi: 10.1016/j.invent.2016.10.002] [Medline: $\underline{30135818]}$

17. Kharrazi H, Lu AS, Gharghabi F, Coleman W. A scoping review of health game research: past, present, and future. Games Health J 2012 Apr 18;1(2):- [FREE Full text] [doi: 10.1089/g4h.2012.0011] [Medline: 24416638]

18. DeSmet A, Shegog R, van Ryckeghem D, Crombez G, de Bourdeaudhuij I. A systematic review and meta-analysis of interventions for sexual health promotion involving serious digital games. Games Health J 2015 Apr;4(2):78-90. [doi: 10.1089/g4h.2014.0110] [Medline: 26181801]

19. Larsen LH, Schou L, Lund HH, Langberg H. The physical effect of exergames in healthy elderly-a systematic review. Games Health J 2013 Aug;2(4):205-212. [doi: 10.1089/g4h.2013.0036] [Medline: 26192224]

20. Proença JP, Quaresma C, Vieira P. Serious games for upper limb rehabilitation: a systematic review. Disabil Rehabil Assist Technol 2018 Jan;13(1):95-100. [doi: 10.1080/17483107.2017.1290702] [Medline: 28359181]

21. Theng Y, Lee JW, Patinadan PV, Foo SS. The use of videogames, gamification, and virtual environments in the self-management of diabetes: a systematic review of evidence. Games Health J 2015 Oct;4(5):352-361. [doi: 10.1089/g4h.2014.0114] [Medline: 26287926]

22. Thomas TH. Protocol and search strategy for a scoping review of serious games for patients with chronic illness. Open Science Framework. 2020 Mar. URL: https://mfr.osf.io/render?url=https://osf.io/k7sy5/ ?direct $\% 26$ mode=render\%26action=download\%26mode=render\%20DOI\%2010.17605/OSF.IO/48ZHJ [accessed 2020-07-15]

23. Tricco AC, Lillie E, Zarin W, O'Brien KK, Colquhoun H, Levac D, et al. PRISMA extension for scoping reviews (PRISMA-ScR): checklist and explanation. Ann Intern Med 2018 Oct 2;169(7):467-473. [doi: 10.7326/M18-0850] [Medline: $\underline{\text { 30178033] }}$

24. About Chronic Diseases. Centers for Disease Control and Prevention. 2019. URL: https://www.cdc.gov/chronicdisease/ about/index.htm [accessed 2020-03-01]

25. Chronic Conditions. Centers for Medicare and Medicaid Services. 2019. URL: https://www.cms.gov/ Research-Statistics-Data-and-Systems/Statistics-Trends-and-Reports/Chronic-Conditions/CC_Main [accessed 2020-07-08]

26. Goodman RA, Posner SF, Huang ES, Parekh AK, Koh HK. Defining and measuring chronic conditions: imperatives for research, policy, program, and practice. Prev Chronic Dis 2013 Apr 25;10:E66 [FREE Full text] [doi: 10.5888/pcd10.120239] [Medline: 23618546]

27. Stokes B. Videogames have changed: time to consider 'serious games'? J Dev Educ 2005;11(3):12 No PMID [FREE Full $\underline{\text { text] }}$

28. Saposnik G, Levin M, Outcome Research Canada (SORCan) Working Group. Virtual reality in stroke rehabilitation: a meta-analysis and implications for clinicians. Stroke 2011 May;42(5):1380-1386. [doi: 10.1161/STROKEAHA.110.605451] [Medline: 21474804] 
29. Burdea G, Polistico K, Krishnamoorthy A, House G, Rethage D, Hundal J, et al. Feasibility study of the BrightBrainer integrative cognitive rehabilitation system for elderly with dementia. Disabil Rehabil Assist Technol 2015;10(5):421-432 [FREE Full text] [doi: 10.3109/17483107.2014.900575] [Medline: 24679074]

30. House G, Burdea G, Polistico K, Grampurohit N, Roll D, Damiani F, et al. A rehabilitation first-tournament between teams of nursing home residents with chronic stroke. Games Health J 2016 Feb;5(1):75-83 [FREE Full text] [doi: 10.1089/g4h.2015.0072] [Medline: 26741697]

31. House G, Burdea G, Polistico K, Roll D, Kim J, Grampurohit N, et al. Integrative rehabilitation of residents chronic post-stroke in skilled nursing facilities: the design and evaluation of the BrightArm duo. Disabil Rehabil Assist Technol 2016 Nov;11(8):683-694. [doi: 10.3109/17483107.2015.1068384] [Medline: 26218427]

32. Kannan L, Vora J, Bhatt T, Hughes SL. Cognitive-motor exergaming for reducing fall risk in people with chronic stroke: a randomized controlled trial. NeuroRehabilitation 2019;44(4):493-510. [doi: 10.3233/NRE-182683] [Medline: 31256084]

33. Simmons CD, Arthanat S, Macri VJ. Pilot study: computer-based virtual anatomical interactivity for rehabilitation of individuals with chronic acquired brain injury. J Rehabil Res Dev 2014;51(3):377-390 [FREE Full text] [doi: 10.1682/JRRD.2013.05.0103] [Medline: 25019661]

34. van Balkom TD, Berendse HW, van der Werf YD, Twisk JW, Zijlstra I, Hagen RH, et al. COGTIPS: a double-blind randomized active controlled trial protocol to study the effect of home-based, online cognitive training on cognition and brain networks in Parkinson's disease. BMC Neurol 2019 Jul 31;19(1):179 [FREE Full text] [doi: 10.1186/s12883-019-1403-6] [Medline: 31366395]

35. Kerfoot BP, Gagnon DR, McMahon GT, Orlander JD, Kurgansky KE, Conlin PR. A team-based online game improves blood glucose control in veterans with type 2 diabetes: a randomized controlled trial. Diabetes Care 2017 Sep;40(9):1218-1225. [doi: 10.2337/dc17-0310] [Medline: 28790131]

36. Gorini A, Marzorati C, Casiraghi M, Spaggiari L, Pravettoni G. A neurofeedback-based intervention to reduce post-operative pain in lung cancer patients: study protocol for a randomized controlled trial. JMIR Res Protoc 2015 May 4;4(2):e52 [FREE Full text] [doi: 10.2196/resprot.4251] [Medline: 25940965]

37. Kim HJ, Kim SM, Shin H, Jang J, Kim YI, Han DH. A mobile game for patients with breast cancer for chemotherapy self-management and quality-of-life improvement: randomized controlled trial. J Med Internet Res 2018 Oct 29;20(10):e273 [FREE Full text] [doi: 10.2196/jmir.9559] [Medline: 30578205]

38. Wang H, McMillan SC, Vijayakumar N, McDonald S, Huang L, Gwede C, et al. A behavioral physical activity intervention to manage moderate and severe fatigue among head and neck cancer patients-pre-efficacy study in the national institutes of health orbit model. Cancer Nurs 2019;42(1):E1-14. [doi: 10.1097/NCC.0000000000000568] [Medline: 29461282]

39. Radhakrishnan K, Toprac P, O'Hair M, Bias R, Kim MT, Bradley P, et al. Interactive digital e-health game for heart failure self-management: a feasibility study. Games Health J 2016 Dec;5(6):366-374 [FREE Full text] [doi: 10.1089/g4h.2016.0038] [Medline: 27976955]

40. Fortunato M, Harrison J, Oon AL, Small D, Hilbert V, Rareshide C, et al. Remotely monitored gamification and social incentives to improve glycemic control among adults with uncontrolled type 2 diabetes (iDiabetes): protocol for a randomized controlled trial. JMIR Res Protoc 2019 Nov 20;8(11):e14180 [FREE Full text] [doi: 10.2196/14180] [Medline: $\underline{31746765]}$

41. Kurtzman GW, Day SC, Small DS, Lynch M, Zhu J, Wang W, et al. Social incentives and gamification to promote weight loss: the LOSE IT randomized, controlled trial. J Gen Intern Med 2018 Oct;33(10):1669-1675 [FREE Full text] [doi: 10.1007/s11606-018-4552-1] [Medline: 30003481]

42. Lyons EJ, Baranowski T, Basen-Engquist KM, Lewis ZH, Swartz MC, Jennings K, et al. Testing the effects of narrative and play on physical activity among breast cancer survivors using mobile apps: study protocol for a randomized controlled trial. BMC Cancer 2016 Mar 9;16:202 [FREE Full text] [doi: 10.1186/s12885-016-2244-y] [Medline: 26960972]

43. Höchsmann C, Infanger D, Klenk C, Königstein K, Walz SP, Schmidt-Trucksäss A. Effectiveness of a behavior change technique-based smartphone game to improve intrinsic motivation and physical activity adherence in patients with type 2 diabetes: randomized controlled trial. JMIR Serious Games 2019 Feb 13;7(1):e11444 [FREE Full text] [doi: 10.2196/11444] [Medline: 30758293]

44. Höchsmann C, Müller O, Ambühl M, Klenk C, Königstein K, Infanger D, et al. Novel smartphone game improves physical activity behavior in type 2 diabetes. Am J Prev Med 2019 Jul;57(1):41-50. [doi: 10.1016/j.amepre.2019.02.017] [Medline: 31128953]

45. Adie K, Schofield C, Berrow M, Wingham J, Humfryes J, Pritchard C, et al. Does the use of Nintendo Wii Sports improve arm function? Trial of Wii in Stroke: a randomized controlled trial and economics analysis. Clin Rehabil 2017 Feb;31(2):173-185. [doi: 10.1177/0269215516637893] [Medline: 26975313]

46. Adomavičienė A, Daunoravičienė K, Kubilius R, Varžaitytė L, Raistenskis J. Influence of new technologies on post-stroke rehabilitation: a comparison of armeo spring to the kinect system. Medicina (Kaunas) 2019 Apr 9;55(4):- [FREE Full text] [doi: 10.3390/medicina55040098] [Medline: 30970655]

47. Choi JH, Han EY, Kim BR, Kim SM, Im SH, Lee SY, et al. Effectiveness of commercial gaming-based virtual reality movement therapy on functional recovery of upper extremity in subacute stroke patients. Ann Rehabil Med 2014 Aug;38(4):485-493 [FREE Full text] [doi: 10.5535/arm.2014.38.4.485] [Medline: 25229027] 
48. Choi Y, Ku J, Lim H, Kim YH, Paik N. Mobile game-based virtual reality rehabilitation program for upper limb dysfunction after ischemic stroke. Restor Neurol Neurosci 2016 May 2;34(3):455-463. [doi: 10.3233/RNN-150626] [Medline: 27163250]

49. da Silva Alves R, Iunes DH, de Carvalho JM, Menezes FD, Silva AM, Borges JB, et al. Effects of exergaming on quality of life in cancer patients. Games Health J 2018 Dec;7(6):385-392. [doi: 10.1089/g4h.2017.0174]

50. da Silva Alves R, Iunes DH, Pereira IC, Borges JB, Nogueira DA, Silva AM, et al. Influence of exergaming on the perception of cancer-related fatigue. Games Health J 2017 Apr;6(2):119-126. [doi: 10.1089/g4h.2016.0051] [Medline: 28418751]

51. da Silva Ribeiro NM, Ferraz DD, Pedreira E, Pinheiro I, da Silva Pinto AC, Neto MG, et al. Virtual rehabilitation via Nintendo Wii and conventional physical therapy effectively treat post-stroke hemiparetic patients. Top Stroke Rehabil 2015 Aug;22(4):299-305. [doi: 10.1179/1074935714Z.0000000017] [Medline: 26258455]

52. Fan S, Su F, Chen S, Hou W, Sun J, Chen K, et al. Improved intrinsic motivation and muscle activation patterns in reaching task using virtual reality training for stroke rehabilitation: a pilot randomized control trial. J Med Biol Eng 2014;34(4):399-407. [doi: 10.5405/jmbe.1502]

53. Gamito P, Oliveira J, Coelho C, Morais D, Lopes P, Pacheco J, et al. Cognitive training on stroke patients via virtual reality-based serious games. Disabil Rehabil 2017 Feb;39(4):385-388. [doi: 10.3109/09638288.2014.934925] [Medline: 25739412]

54. Hickman RL, Clochesy JM, Pinto MD, Burant C, Pignatiello G. Impact of a serious game for health on chronic disease self-management: preliminary efficacy among community dwelling adults with hypertension. J Health Hum Serv Adm 2015;38(2):253-275. [Medline: 26442364]

55. Hung J, Chou C, Chang H, Wu W, Hsieh Y, Chen P, et al. Cognitive effects of weight-shifting controlled exergames in patients with chronic stroke: a pilot randomized comparison trial. Eur J Phys Rehabil Med 2017 Oct;53(5):694-702. [doi: 10.23736/S1973-9087.17.04516-6] [Medline: 28382812]

56. Kempf K, Martin S. Autonomous exercise game use improves metabolic control and quality of life in type 2 diabetes patients - a randomized controlled trial. BMC Endocr Disord 2013 Dec 10;13:57 [FREE Full text] [doi: 10.1186/1472-6823-13-57] [Medline: 24321337]

57. Kim SM, Kim H, Hwang HC, Hong JS, Bae S, Min KJ, et al. The effects of a serious game on depressive symptoms and anxiety in breast cancer patients with depression: a pilot study using functional magnetic resonance imaging. Games Health J 2018 Dec;7(6):409-417. [doi: 10.1089/g4h.2017.0183] [Medline: 30383458]

58. Klompstra L, Jaarsma T, Strömberg A. An in-depth, longitudinal examination of the daily physical activity of a patient with heart failure using a Nintendo Wii at home: a case report. J Rehabil Med 2013 Jun;45(6):599-602 [FREE Full text] [doi: 10.2340/16501977-1151] [Medline: 23695704]

59. Senior H, Henwood T, de Souza D, Mitchell G. Investigating innovative means of prompting activity uptake in older adults with type 2 diabetes: a feasibility study of exergaming. J Sports Med Phys Fitness 2016 Oct;56(10):1221-1225. [Medline: 26329840]

60. Shin J, Bog Park S, Ho Jang S. Effects of game-based virtual reality on health-related quality of life in chronic stroke patients: a randomized, controlled study. Comput Biol Med 2015 Aug;63:92-98. [doi: 10.1016/j.compbiomed.2015.03.011] [Medline: 26046499]

61. Shin J, Kim M, Lee J, Jeon Y, Kim S, Lee S, et al. Effects of virtual reality-based rehabilitation on distal upper extremity function and health-related quality of life: a single-blinded, randomized controlled trial. J Neuroeng Rehabil 2016 Feb 24;13:17 [FREE Full text] [doi: 10.1186/s12984-016-0125-x] [Medline: 26911438]

62. Şimşek TT, Çekok K. The effects of Nintendo Wii(TM)-based balance and upper extremity training on activities of daily living and quality of life in patients with sub-acute stroke: a randomized controlled study. Int J Neurosci 2016 Dec;126(12):1061-1070. [doi: 10.3109/00207454.2015.1115993] [Medline: 26626539]

63. Song GB, Park EC. Effect of virtual reality games on stroke patients' balance, gait, depression, and interpersonal relationships. J Phys Ther Sci 2015 Jul;27(7):2057-2060 [FREE Full text] [doi: 10.1589/jpts.27.2057] [Medline: 26311925]

64. Sutanto Y, Makhabah D, Aphridasari J, Doewes M, Suradi, Ambrosino N. Videogame assisted exercise training in patients with chronic obstructive pulmonary disease: a preliminary study. Pulmonology 2019;25(5):275-282 [FREE Full text] [doi: 10.1016/j.pulmoe.2019.03.007] [Medline: $\underline{\text { 31076287] }}$

65. Towle D, Edmans J, Lincoln N. Use of computer-presented games with memory-impaired stroke patients. Clin Rehabil 2016 Jul;2(4):303-307. [doi: 10.1177/026921558800200406]

66. van de Weijer SC, Duits AA, Bloem BR, Kessels RP, Jansen JF, Köhler S, et al. The Parkin'Play study: protocol of a phase II randomized controlled trial to assess the effects of a health game on cognition in Parkinson's disease. BMC Neurol 2016 Nov 3;16(1):209 [FREE Full text] [doi: 10.1186/s12883-016-0731-z] [Medline: 27809791]

67. van Santen J, Dröes RM, Bosmans JE, Blanson Henkemans OA, van Bommel S, Hakvoort E, et al. The (cost-) effectiveness of exergaming in people living with dementia and their informal caregivers: protocol for a randomized controlled trial. BMC Geriatr 2019 Feb 19;19(1):50 [FREE Full text] [doi: 10.1186/s12877-019-1062-x] [Medline: 30782127 ]

68. Yu F, Mathiason MA, Johnson K, Gaugler JE, Klassen D. Memory matters in dementia: efficacy of a mobile reminiscing therapy app. Alzheimer's Dement (N Y) 2019;5:644-651 [FREE Full text] [doi: 10.1016/j.trci.2019.09.002] [Medline: $\underline{31720365]}$ 
69. Abraham O, LeMay S, Bittner S, Thakur T, Stafford H, Brown R. Investigating serious games that incorporate medication use for patients: systematic literature review. JMIR Serious Games 2020 Apr 29;8(2):e16096 [FREE Full text] [doi: 10.2196/16096] [Medline: 32347811 ]

70. Klonoff DC. Behavioral theory: the missing ingredient for digital health tools to change behavior and increase adherence. J Diabetes Sci Technol 2019 Mar;13(2):276-281 [FREE Full text] [doi: 10.1177/1932296818820303] [Medline: 30678472]

71. Rohrbach N, Chicklis E, Levac DE. What is the impact of user affect on motor learning in virtual environments after stroke? A scoping review. J Neuroeng Rehabil 2019 Jun 27;16(1):79 [FREE Full text] [doi: 10.1186/s12984-019-0546-4] [Medline: $\underline{31248439}]$

72. Verschueren S, Buffel C, vander Stichele G. What is the impact of user affect on motor learning in virtual environments after stroke? A scoping review. JMIR Serious Games 2019 May 2;7(2):e11565 [FREE Full text] [doi: 10.2196/11565] [Medline: $\underline{31045496]}$

73. Cugelman B. Gamification: what it is and why it matters to digital health behavior change developers. JMIR Serious Games 2013 Dec 12;1(1):e3 [FREE Full text] [doi: 10.2196/games.3139] [Medline: 25658754]

74. Kuipers DA, Terlouw G, Wartena BO, van 't Veer JT, Prins JT, Pierie JPE. The role of transfer in designing games and simulations for health: systematic review. JMIR Serious Games 2017 Nov 24;5(4):e23 [FREE Full text] [doi: 10.2196/games.7880] [Medline: 29175812]

\begin{abstract}
Abbreviations
COPD: chronic obstructive pulmonary disease

$\mathbf{H b A}_{1 \mathbf{c}}$ : hemoglobin $\mathrm{A}_{1 \mathrm{c}}$
\end{abstract}

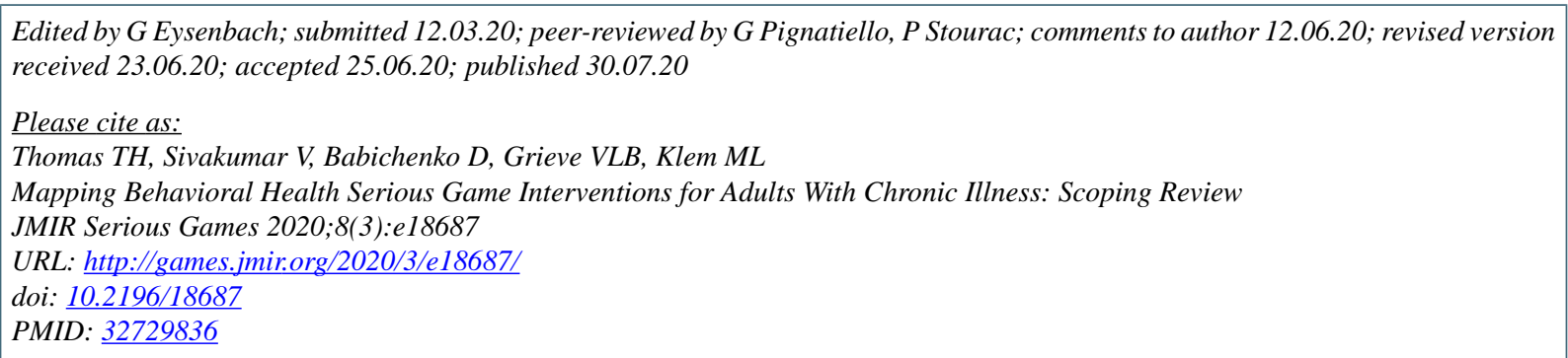

(C) Teresa Hagan Thomas, Varshini Sivakumar, Dmitriy Babichenko, Victoria L B Grieve, Mary Lou Klem. Originally published in JMIR Serious Games (http://games.jmir.org), 30.07.2020. This is an open-access article distributed under the terms of the Creative Commons Attribution License (https://creativecommons.org/licenses/by/4.0/), which permits unrestricted use, distribution, and reproduction in any medium, provided the original work, first published in JMIR Serious Games, is properly cited. The complete bibliographic information, a link to the original publication on http://games.jmir.org, as well as this copyright and license information must be included. 